$10 \mid 2021$

Intelligence artificielle, pratiques sociales et politiques publiques

\title{
Les sciences humaines et sociales (SHS) et les sciences de l'information et de la communication (SIC) aux défis de l'IA
}

Human and social sciences (SHS) and information and communication sciences

(CIS) to the challenges of AI

Las ciencias humanas y sociales (SHS) y las ciencias de la información y la comunicación (CIS) a los desafíos de la IA

\section{Laurent Petit}

\section{(2) OpenEdition}

\section{Journals}

Édition électronique

URL : https://journals.openedition.org/ctd/4475

DOI : $10.4000 /$ ctd. 4475

ISSN : 2491-1437

\section{Éditeur}

Chaire Unesco Pratiques émergentes en technologies et communication pour le développement

\section{Édition imprimée}

ISBN : 2491-1437

\section{Référence électronique}

Laurent Petit, «Les sciences humaines et sociales (SHS) et les sciences de l'information et de la communication (SIC) aux défis de l'IA », Communication, technologies et développement [En ligne], 10 | 2021, mis en ligne le 20 mai 2020, consulté le 27 mai 2021. URL : http://journals.openedition.org/ctd/ 4475 ; DOI : https://doi.org/10.4000/ctd.4475

Ce document a été généré automatiquement le 27 mai 2021

Communication, technologies et développement 


\section{Les sciences humaines et sociales (SHS) et les sciences de l'information et de la communication (SIC) aux défis de l'IA}

Human and social sciences (SHS) and information and communication sciences (CIS) to the challenges of AI Las ciencias humanas y sociales (SHS) y las ciencias de la información y la comunicación (CIS) a los desafíos de la IA

Laurent Petit

1 Les espoirs suscités par l'IA ne sont pas nouveaux tant la recherche dans ce domaine s'est fait une spécialité d'avancer, davantage que dans d'autres domaines, par prophéties auto-réalisatrices (Merton, 1948), la plus célèbre étant la défaite de Garry Kasparov, champion du monde d'échecs contre Deep Blue en 1997. Mais de nombreuses promesses n'ont été purement et simplement pas tenues (Jordan, 2018), contribuant ainsi à alimenter une abondante littérature de science-fiction qui suscite autant d'espoirs que de peurs.

\section{Les SHS chahutées par l'IA}

2 Il n'en est pas moins vrai que l'intelligence artificielle semble connaitre depuis quelques années un emballement médiatique déjà rencontré pour la cybernétique ${ }^{1}$ après-guerre par exemple, emballement dont il conviendrait d'établir la genèse. Nous ne la ferons pas ici, elle nécessiterait un programme de recherche en elle-même. Contentons-nous à ce stade d'opérer ce rapprochement à titre d'hypothèse. Ronan Le Roux (2018) montre, s'agissant de l'histoire de la notion en France de 1948 à 1975, que 
la modélisation cybernétique à l'œuvre dans les sciences biologiques, humaines et sociales recouvre des «façons de penser » disparates. Ni paradigme ni épistémè selon l'auteur et, cependant, une propension à se présenter - comme l'intelligence artificielle plus tard ? - comme « référence unificatrice » ou « bannière fédératrice ».

\section{IA connexionniste ou la révolution annoncée}

3 Cette hypothèse se trouve alimentée par le fait que l'IA, qui est tout sauf un champ homogène, semble aujourd'hui, dans ses développements les plus récents, consacrer la victoire posthume d'une première vague de l'IA d'inspiration cybernétique appelée connexionniste par rapport à une IA symbolique qui a longtemps dominé le champ.

4 La tension entre ces deux approches est née avec la démarcation que la naissance de l'Intelligence artificielle opposa à la première cybernétique. L'approche symbolique qui constitue le cadre de référence initial de l'IA s'est identifiée à un cognitivisme orthodoxe : penser, c'est calculer des symboles qui ont à la fois une réalité matérielle et une valeur sémantique de représentation. En revanche, le paradigme connexionniste considère que penser s'apparente à un calcul massivement parallèle de fonctions élémentaires - celles qui seront distribuées au sein d'un réseau de neurones - dont les comportements signifiants n'apparaissent au niveau collectif que comme un effet émergent des interactions produites par ces opérations élémentaires (Cardon et al., p. 178, citant Andler, 1992) ${ }^{2}$.

5 Cette approche donne du poids à la thèse d'une science désormais en mesure de tenir la promesse d'une instrumentation des médiations sociales grâce aux progrès représentés par le big data, considéré comme la gestion dynamique de données hétérogènes qui ne se confond pas avec le traitement massif des données (nous reviendrons sur ce point en $2^{\mathrm{e}}$ partie).

6 L'adoption de cette approche, dont nous essaierons ultérieurement de mesurer les conséquences, ne peut se comprendre sans faire référence à un double processus de naturalisation des phénomènes sociaux et de fétichisation du nombre, phénomènes liés, mais qu'il convient à ce stade de distinguer.

\section{Naturalisation des phénomènes sociaux}

7 Un processus de naturalisation est donc à l'œuvre qui n'est ni nouveau ni homogène. Daniel Andler (2016) s'est penché en philosophe sur la place du naturalisme aujourd'hui : ne se situant ni dans la totale adhésion ni la méfiance sans distance, il en propose une analyse critique nuancée en suivant de près plusieurs programmes de recherche dont il examine la capacité à rendre compte du réel. Il fait un focus détaillé sur trois domaines en plein essor, dont la place au sein des sciences est autant louée que redoutée : les sciences cognitives, les neurosciences et la biologie évolutive. Cette approche pointilliste - particulièrement bienvenue et bien documentée - n'occulte pas le fait que ces sciences tentent de prendre une place qui n'était naguère encore pas la leur dans la sphère académique, suscitant une méfiance compréhensible de la part des sciences humaines et sociales qui mettent en œuvre d'autres méthodes sur les mêmes objets.

8 Au-delà du périmètre des sciences au sens strict, ce que nous préférons appeler processus de naturalisation plutôt que naturalisme a gagné des sphères plus 
médiatiques, y compris lorsque les propositions qui en découlent sont le fait de personnalités ayant une formation scientifique, pour beaucoup en biologie. Pour ne prendre qu'un exemple récent, mentionnons le biologiste François Taddei qui, sans citer Pierre Lévy (1994), intègre une touche d'« intelligence collective " pour définir sa " société apprenante »: " Dans une organisation apprenante, tous les membres peuvent apprendre les uns des autres, les expérimentations et les apprentissages des uns facilitant ceux des autres. Les individus comme le collectif sont invités à apprendre à apprendre et à développer des approches réflexives pour faciliter les dynamiques d'apprentissage. Ces échanges transversaux permettent l'émergence de transformations, fondées sur l'intelligence collective et l'adaptation permanente aux changements de l'environnement, assurant le développement durable de l'organisation » (Becchetti-Bizot, Houzel, Taddei, 2017, p. 60).

9 Le processus de "naturalisation » qui s'opère est en fait double: dans le sens d'une tentative de réduction de l'humain à sa dimension biologique comme nous venons de l'évoquer, mais aussi dans le sens d'Yves Jeanneret (2014) de ce qui ne se discute plus. S'il s'agit au départ de simples métaphores, elles ont tendance peu à peu à se transformer en concepts, ne serait-ce que par défaut. Or, ces conceptions sous-jacentes gagnent à être rendues explicites et mises en perspective. Nous reviendrons sur ce point majeur.

\section{Fétichisation du nombre}

10 S'agissant de la fétichisation du nombre, Alain Supiot dans un ouvrage remarquable (2015), tiré de cours donnés au Collège de France de 2012 à 2014, insiste sur la résurgence du scientisme, depuis les années 1980, résurgence qui va de pair « avec la foi ultralibérale en un monde global régi par les lois immanentes de l'économie et peuplé de particules contractantes mues par le calcul de leurs utilités individuelles » (op. cit.). Il montre que ce crédo a donné naissance à une extension du paradigme du marché à toutes les activités humaines. Ces évolutions expliquent selon lui le règne absolu actuel de la quantification ${ }^{3}$ et le passage du gouvernement à la gouvernance, système dans lequel «la quantification sert moins à représenter la réalité qu'à évaluer des performances. » (op. cit.).

11 Pour qualifier les évolutions contemporaines, Alain Supiot (2016, p. 56) parle d'une "opposition qui conduit aujourd'hui, d'une part, au retour du biologisme et à ce qu'Alain Prochiantz a nommé « l'étrange fureur d'être singe » et, d'autre part, au postmodernisme qui, au nom de «l'arbitraire du signe ", prétend a l'inverse désancrer le symbolique du biologique. " En témoignent les «Google sciences » chères à Chris Anderson $(2008)^{4}$, qui prétendent prédire les comportements humains davantage que comprendre les ressorts de ceux-ci, au nom d'un comportementalisme (Pasquale, 2015) issu d'un processus de naturalisation que l'on pourrait considérer comme abouti.

Ce comportementalisme radical tel qu'il ressort des avatars générés par la gouvermentalité algorithmique ${ }^{5}$, entendue comme "un certain type de rationalité (a)normative ou (a)politique reposant sur la récolte, l'agrégation et l'analyse automatisée de données en quantité massive de manière à modéliser, anticiper et affecter par avance les comportements possibles» (Rouvroy, Berns, 2013, p. 173), ne peut être rendu crédible que par un processus de naturalisation préalable. On a affaire ici à un homme «hors sol» ou, pour le dire comme Antoinette Rouvroy et Thomas 
Berns, à un double statistique détaché de nous. Paradoxalement c'est donc au moment où ce détachement se produit que la tentation de la naturalisation des comportements se fait pressante dans nombre de disciplines. A contrario, il nous faut oser requalifier ceux-ci en "faits sociaux ", consistant " en des manières d'agir, de penser et de sentir, extérieures à l'individu, et qui sont douées d'un pouvoir de coercition en vertu duquel ils s'imposent à lui » (Durkheim, 1981, p.5), se distinguant ainsi des phénomènes organiques et des phénomènes psychiques.

\section{Les défis épistémologiques posés par l'IA pour les sciences de la nature et de la culture}

Le défi lancé s'adresse à l'ensemble de la recherche, pas seulement aux sciences humaines et sociales. Avant de nous interroger sur la façon dont il touche les sciences de la culture, il nous faut comprendre comment la révolution du calcul et des big data s'applique aux sciences de la nature.

\section{IA et changement de paradigme}

D’après Bruno Bachimont (2015), la science serait passée d'une épistémologie de la mesure à une épistémologie du calcul ou de la donnée. Il s'attache tout d'abord à montrer comment fonctionne le paradigme de la mesure dans la science dite moderne, entendez dans les sciences de la nature. Quand Bachimont dit que la corrélation a succédé à la causalité ou l'épistémologie de la mesure à l'épistémologie de la donnée, on peut préciser que les études de corrélation existaient évidemment dans le paradigme de la mesure, mais entre des variables en nombre fini et à partir d'hypothèses forgées elles-mêmes à partir de théories.

Dans les sciences de la culture aussi d'ailleurs, même si la recherche de corrélations n'est pas la méthode qui y est la plus répandue. Que l'on songe à Emile Durkheim par exemple, considéré comme le père d'une approche quantitative de la sociologie. $\mathrm{A}$ propos du Suicide (1897) de Durkheim, Jean-Claude Passeron (2001, p. 47) dit que ce dernier, qui a voulu fournir un argumentaire exemplaire de sociologie quantitative, montre pourtant, au fil des arguments, le rôle actif que joue l'interprétation du "sens » des actions dans la construction par Durkheim des « trois types de suicide » (sic)6.

16 Mais l'enjeu ne semble plus si situer ici désormais. Pour prendre la mesure d'une rupture épistémologique rendue possible par un phénomène de numérisation généralisée, il faut en effet éviter de confondre big data, entendue comme la gestion dynamique de données hétérogènes et masse de données, comprise comme la gestion d'un grand nombre de données rendue possible par l'augmentation des capacités de calcul automatisé. La première ne va pas sans l'autre, mais le changement n'est pas que quantitatif.

\section{Rupture ou cohabitation?}

17 Ce mouvement opérerait, selon Bachimont, une triple rupture: une rupture des données par rapport à leur origine et leur nature (la collecte des données reposant désormais sur la captation d'informations d'origines diverses, selon des périodicités élevées, et hétérogènes dans leur nature et dans leur format), une rupture du 
traitement par rapport aux données (traitement qui repose sur l'utilisation d'outils en général mathématiques et statistiques), enfin une rupture de ce qui est montré par rapport à ce qui est calculé (la visualisation des résultats reposant sur la présentation des résultats selon des conventions souvent cartographiques, s'inspirant des techniques de domaine de l'InfoViz ou Information Visualisation). Cette rupture conduirait via un changement de paradigme ${ }^{7}$ à une nouvelle "science des données ».

Si une nouvelle logique s'amorce indubitablement, peut-être ne faudrait-il pas en conclure trop rapidement à un changement de paradigme qui ne se laisse pas observer facilement (Petit, 2008). La fausse monnaie n'a peut-être pas tout à fait chassé la bonne et, comme nous l'avons montré dans le champ de l'éducation, un " entre-deux » peut s'installer durablement.

\section{Le cas de la formation}

19 Nous voudrions illustrer ici le propos par des faits pris dans le champ de l'éducation. Parce que nous pensons pas mal le connaître, ensuite parce qu'il est nécessaire de réaffirmer que, contrairement à une idée reçue, il ne se contente pas de réagir à ou, pour reprendre un anglicisme à la mode, d'être impacté par des phénomènes sociaux qui lui seraient extérieurs. A contrario a-t-il souvent constitué un champ d'expérimentation d'innovations qui se sont diffusées ensuite à plus grande échelle, comme l'a montré Pierre Mœglin pour le satellite éducatif (1994).

\section{Des études expérimentales non signifiantes}

Dans le champ éducatif, on y a de longue date tenté l'approche expérimentale sous la forme d'essais randomisés pour tenter de mesurer l'efficacité d'une pratique comparée à une autre. Thomas Russell (2001) a collecté près de 2500 études comparant depuis 1928 les résultats des étudiants ayant suivi des cours en présentiel et ceux d'étudiants ayant suivi des cours par correspondance et à distance. Le résultat de ses investigations est visible sur un site au nom explicite: NSDP pour «No Significant Difference Phenomenon $^{8}$ ». Dans le panel étudié, les modalités des cours à distance sont variées, qu'il s'agisse de documents imprimés envoyés aux étudiants, d'émissions de radio ou de télévision, de vidéos ou de toute autre ressource en ligne. La majorité de ces études portant sur l'efficacité de l'usage d'outils ou de médias dans la formation à distance ne montrent pas d'améliorations significatives des élèves. Faut-il en conclure qu'il ne passe rien? Pas nécessairement. Peut-être faut-il alors questionner ce que des évaluations de ce type évaluent et les compléter par d'autres méthodes si l'on veut mesurer, non seulement ce que les élèves ont accompli, mais la manière dont ils ont mené leur apprentissage. Bref, aller vers des méthodes propres aux sciences de l'interprétation (Passeron, 2001), comme nous l'avons naguère tenté dans une expérience à grande échelle visant à rendre plus interactifs les enseignements en amphithéâtre (Lamine, Petit, 2014).

21 Ces résultats édifiants, le plus souvent ignorés, n'arrêtent en rien les zélotes de la «scientifisation» des recherches en éducation qui tentent, forts de l'avancée des connaissances dans certaines disciplines, de relancer à nouveau frais la méthode expérimentale là où elle n'a pas fait ses preuves, tout en l'affublant de nouvelles approches qui s'inscrivent en rupture avec celle-ci. 


\section{Des sciences de la nature désormais outillées} justifie ici l'irruption de sciences de la nature outillées par l'informatique dans un champ où elles n'étaient pas légitimes, en tout cas sur un terrain traditionnellement occupé par d'autres. Une alliance objective sellée entre les sciences de gestion, les sciences cognitives et l'informatique semble désormais en mesure de s'engager dans une instrumentation des médiations sociales. Dans l'éducation, elle prétend renouveler la question (déjà ancienne) de l'industrialisation via les promesses de l'algorithmisation de la formation, notamment sur le sujet sensible de l'individualisation. L'échec relatif des MOOC - au regard des ambitions initiales affichées tout au moins - tient en partie selon nous à une promesse non tenue sur cette question essentielle. Comment proposer à chaque inscrit un parcours qui lui soit propre (au-delà de quelques parcours-types correspondant à des profils définis a priori par les concepteurs) et surtout comment assurer un suivi et un accompagnement qui ne se limite pas à la possibilité d'échanger avec ses pairs sur un forum en ligne ? Or, on sait depuis fort longtemps que là réside la clé de la réussite en enseignement à distance, connu pour ses taux d'abandon élevés, taux que l'on retrouve peu ou prou dans les MOOC'.

\section{Automatiser l'individualisation ?}

Dans le passé, le psychologue américain B. F. Skinner (1904-1990) a cherché à promouvoir un type d'industrialisation cherchant à concilier massification et individualisation au nom d'une augmentation de la productivité de l'enseignement. «Par là il prend le contre-pied de W. Schramm et des responsables de programmes, à l'Unesco et dans d'autres organisations nationales et internationales, qui, dans les années 1960 et 1970, lancent des réseaux de radio et de télévision pour acheminer les meilleurs cours au plus grand nombre possible d'élèves ${ }^{10}$.» (Mœglin, 2016, p. 84). Ces tentatives ont fait long feu malgré l'importance des moyens mis à disposition aux EtatsUnis. Pour expliquer cet échec, Pierre Mœglin met en avant deux raisons : en premier lieu, l'inspiration behaviouriste de cet enseignement programmé a été fortement critiquée notamment par les courants cognitivistes, constructivistes ou socioconstructiviste de la pédagogie. En second lieu, les moyens techniques de l'époque étaient trop frustres pour permettre une individualisation des rythmes d'apprentissage qui permette une véritable diversification des contenus et des parcours.

Sautons quelques décennies et voyons comment l'intelligence artificielle est susceptible aujourd'hui de revisiter ces deux points. S'agissant de l'approche pédagogique, les discours contemporains mettent l'accent sur la notion d'individualisation. Dans l'attente d'investigations plus poussées, Antoinette Rouvroy et Thomas Berns nous invitent à bien marquer la différence entre personnalisation et individualisation. Selon eux, l'algorithmisation permettrait d'améliorer la personnalisation de l'offre, du service proposé à partir de besoins non exprimés, « remontés » en toute objectivité à partir de données ou plutôt de traces presque laissées par l'individu à son corps défendant, sans l'écran de la conscience du sujet pourrait-on dire : cela revient à

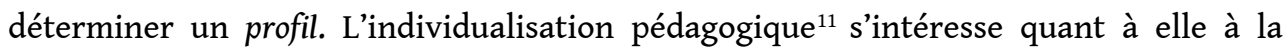
réalité de l'individu dans sa complexité et a pour ambition de le faire grandir, progresser: il y a là un projet émancipateur qui ne saurait se résumer à une

Communication, technologies et développement, $10 \mid 2021$ 
"segmentation de marché » (Rouvroy, Berns, 2013, p. 176). S'agissant des progrès techniques, s'ils sont indéniables, sont-ils suffisants pour espérer - enfin - atteindre cette individualisation automatisée tant espérée dans la formation?

\section{Comment les SHS peuvent-elles répondre?}

25 A l'issue de ces quelques remarques, on mesure l'ampleur du défi lancé aux SHS alors qu'elles n'ont jamais été autant disqualifiées (dans les discours et les moyens accordés). Une déconstruction des discours prophétiques et une mise au jour des enjeux de l'effet de saturation qu'ils imposent dans l'espace public s'imposent. Mais cela se révélera-t-il suffisant?

\section{Un construit social à questionner, et après ?}

Une première réponse semble en effet aller de soi tant elle constitutive de nombreuses disciplines, des sciences de l'information et de la communication en premier lieu : elles sont armées pour discuter les notions, les mettre en perspective, opérer une déconstruction des discours, bref considérer ces avancées comme un construit social, mais en se préservant de la critique du relativisme faite à Bruno Latour lorsqu'il est penché sur le fonctionnement des laboratoires de recherche (La science en action, 2005). Il s'agit pour cela de mettre en perspective les circonstances et les jeux d'acteurs qui ont présidé à leur construction (Bourdieu parle de défatalisation; ni hasard ni nécessité). Mais il ne nous semble pas adéquat de parler ici de déconstruction (qui s'apparente trop à une critique radicale dans le mauvais sens du terme, celle qui détruit, mais ne produit rien). Il s'agit plutôt de donner à voir et à comprendre, comme un éclaté ou un écorché.

Mais cette opération ne saurait suffire. Nous sommes en effet confrontés à une difficulté majeure: comment réintroduire de l'interprétation lorsque nous ne maitrisons pas les modalités de construction de la donnée ? Avec la révolution annoncée des big data, les sciences de la culture se mettraient au diapason des sciences de la nature : la corrélation statistique établie à partir du traitement d'un nombre considérable de données révèlerait en quelque sorte un sens caché, débarrassé des oripeaux idéologiques de certaines disciplines. La recherche ne serait plus tant la construction d'un discours par l'interprétation basé sur des faits, des comparaisons, des enquêtes, etc. que la découverte - « objective » - de réalités jusqu'ici cachées : faire de la recherche sur les faits sociaux pourrait être assimilé à une sorte de dévoilement, ne donnant pas prise à la subjectivité du chercheur.

Mais «... entre la puissance d'agir fondée sur la corrélation précise, mais aveugle et l'intelligibilité qui permet de comprendre sans permettre l'efficacité décisionnelle et prédictive » (René Thom, 2009) ${ }^{12}$, est-il permis de ne pas choisir ? Les SHS ont une place légitime à prendre pourvu qu'elles ne se laissent pas enfermer dans cette alternative.

\section{Un partenariat équilibré à construire}

Cette place ne peut se trouver qu'avec les autres sciences dans un partenariat scientifique aussi ardu que potentiellement fécond. S'il ne faut pas prendre pour argent comptant les dires de Anderson sur la «Google science ", il ne s'agit pas de contester 
que les big data (et l'IA) interviennent en modifiant la façon de faire de la science. JeanGabriel Ganascia (2019) nous y incite lorsqu'il évoque la valeur heuristique des calculs automatisés menés en dehors de tout modèle théorique. Ils peuvent en effet servir à forger des hypothèses qu'il s'agit ensuite de mettre à l'épreuve, dans l'exemple pris par Ganascia par la démarche expérimentale - Claude Bernard pour la médecine -, mais aussi, nous insistons sur ce point, par les sciences de l'interprétation. En somme, les SHS ne doivent pas se cantonner à un discours sûr, mais, fort de leurs acquis et de leurs méthodes, participer pleinement à l'analyse de ces nouveaux jeux de données. Dès lors ce que la « Google science » que Anderson appelle de ses vœux ne serait au mieux qu'un moment du raisonnement scientifique (Passeron, 2001) qui devrait ensuite faire intervenir d'autres approches avec d'autres méthodes, pour autant que l'on cherche à comprendre et pas seulement à prédire. Il faut pour cela accepter l'éclectisme méthodologique et le dialogue interdisciplinaire, sinon les avancées du deep learning risquent de mener à des impasses, comme le souligne Ganascia. Bref, il nous semble évident que les SHS ont autant à apprendre de l'IA que l'inverse. C'est donc un dialogue paritaire, établi sur un pied d'égalité qu'il convient d'instaurer.

Pour cela, inutile d'inventer de nouvelles catégories. Signalons à cet égard l'ambivalence - pour ne pas dire l'ambiguïté - de la notion d'«humanités numériques »: s'agit-il de donner à analyser les faits humains par des sciences du numérique ou bien d'humaniser au sens fort du terme des sciences qui traditionnellement ne traitent pas de l'homme en société ? Mais pour n'en être que les auxiliaires ou les supplétifs en acceptant par exemple d'être cantonnés à l'ajout d'une coloration éthique? En revanche, oui s'il s'agit d'humaniser le numérique, au sens d'y introduire le recul et l'approche critique propres aux SHS. En d'autres termes, les avancées de l'intelligence artificielle nous offrent une occasion unique de repenser les conditions de possibilité de la présomption (plus que de la preuve), au sein des SHS, des SIC en premier lieu.

\section{Quelles pistes pour l'avenir?}

31 Dans un travail interdisciplinaire exigeant, il y a lieu donc de s'associer avec des spécialistes de l'IA afin d'établir un programme de recherche commun mettant au centre de son questionnement la notion fondamentale de l'apprentissage, de la machine et de l'humain, même si nous ne voulons pas laisser croire à un parallélisme trop évident. Nous faisons l'hypothèse que la question de l'apprentissage humain, activité sociale s'il en est, ne sera pas réglée uniquement par une meilleure compréhension des mécanismes du cerveau. Mais il nous faut comprendre suffisamment comment fonctionne le machine learning, au cœur des préoccupations actuelles des spécialistes de l'IA, pour en être en mesure d'en analyser la portée et les enjeux pour l'apprentissage humain, champ qui pourrait bien être à l'avant-garde de l'innovation comme il le fut naguère avec les satellites éducatifs (Mœglin, 1994).

\section{En comprendre suffisamment sur les avancées de l'IA en général}

La difficulté est réelle eu égard à la technicité de l'entreprise et au fait que les procédures d'apprentissage artificiel sont nombreuses, même si les réseaux de neurones en ce moment le vent en poupe ${ }^{13}$. Il y a lieu de s'y intéresser sérieusement en 
s'attachant le concours d'experts capables d'en présenter simplement les principes de fonctionnement.

Comme le soulignaient déjà Antoinette Rouvroy et Thomas Berns (op. cit., p. 166), " dès lors que les machines deviennent de plus en plus "autonomes » et "intelligentes", elles restent bien sûr dépendantes de leur design initial, des intentions, scripts ou scenarii en fonction desquels elles ont été imaginées. Elles sont, dès leur conception (et quelles que soient les formes qu'elles prennent ensuite), porteuses des visions du monde, attentes et projections conscientes ou inconscientes de leurs concepteurs ».

Mais avec les nouvelles formes de l'intelligence artificielle, mettre au jour ces visions du monde devient de moins en moins aisé. Jean-Sébastien Vayre (2018), s'intéressant au cas des technologies d'apprentissage artificiel appliquées à la gestion de la relation client, montre que, dans le cas de réseaux de neurones artificiels, elles recouvrent un travail de conception de trois cadres sociocognitifs (l'environnement d'apprentissage, l'environnement de traitement et l'environnement politique) qui orientent les formes de la socialisation algorithmique des machines par les humains. "Autrement dit, ce biais d'apprentissage prend la forme concrète d'un ensemble d'opérateurs de traduction qui transforment les régularités que représentent les big data en des informations qui doivent autoriser l'automatisation de la communication organisationnelle.» (op.cit., p. 109). Une des difficultés réside dans le fait que, dans chacun de ces cadres, le " concepteur » et l'« implémenteur » (très humains) y jouent des rôles différents qu'il convient d'analyser empiriquement et finement. La tâche n'est pas aisée sans un bagage technique minimal ou s'en s'adjoindre des compétences rarement rencontrées dans nos disciplines.

\section{Un focus sur la question de la personnalisation en éducation}

Qu'en est-il de cet « avènement d'une société de services véritablement personnalisés (Kohler \& Weisz, 2016)» (Vayre, 2018, p. 95) dans l'éducation? L'apprentissage adaptatif ou adaptive learning ne prétend-il pas aller au-delà d'un simple profilage des apprenants. Le site officiel Eduscol, reprenant l'article de Wikipedia, le définit comme "une méthode pédagogique basée sur l'usage des ordinateurs comme dispositifs d'enseignement interactifs, pour gérer l'attribution de ressources (numériques ou non) en fonction des besoins propres à chaque apprenant. Les ordinateurs adaptent les ressources pédagogiques selon les besoins d'apprentissage des élèves, identifiés grâce aux réponses à des questions, des exercices et des expériences. Cette technologie se base sur différents champs d'études tels que l'informatique, l'éducation, la psychologie et les neurosciences. »

Les effets se font déjà sentir puisque, dans le cadre du partenariat d'innovation et intelligence artificielle (P2IA), six «solutions» d'aide à la décision pour la différenciation et la personnalisation des apprentissages grâce à des solutions innovantes basées sur l'intelligence artificielle (IA)» viennent d'être retenues pour l'apprentissage du français et des mathématiques en cycle 2.

Sur le plan scientifique, les travaux du courant travaillant sur les Learning analytics serait à analyser de près. Vanda Luengo dans un entretien avec Daniel Peraya pour la revue Distances et Médiations des savoirs (2019) distingue le Data mining des Learning analytics. Le premier produit des connaissances à partir de données sans pour autant s'affranchir de la théorie: «il permet d'identifier des phénomènes que l'on n'avait 
jamais pu observer auparavant et que les théories permettent ensuite d'expliquer et de comprendre » (ibid.). Dans le second cas, il s'agit selon Vanda Luengo "d'une approche plutôt explicative permettant la prise de décision » (ibid.). Dans ces tentatives, elle reconnait que des "données épaisses ", c'est-à-dire qui décrivent l'activité de l'enseignant de façon plus fine que des statistiques de connexion par exemple, sont nécessaires et admet que les données recueillies ont plus d'épaisseur dans un EIAH que celles que l'on peut récolter dans un $\mathrm{MOOC}$, «sans doute car les premiers sont plus orientés vers l'apprentissage et vers la recherche que vers la diffusion à grande échelle : leur ingénierie est donc différente » (ibid.). Ou dit autrement, parce qu'il est peut-être plus facile de croiser des données multiples dans un système fermé dont on connaît mieux a priori les principes de conception? Vanda Luengo travaillant à l'adaptation et à la personnalisation de l'apprentissage, considère que les Learning analytics peuvent "être considérées comme une des technologies qui peuvent grandement contribuer à cette transformation de la posture de l'enseignant » (ibid.), accompagnateur du processus d'apprentissage plus que transmetteur de connaissances.

L'évolution de ce courant en informatique nous semble d'ailleurs révélatrice d'un processus d'accumulation et de croisement de méthodes (davantage que de leur substitution) : celles des Learning analytics s'inscrivant dans la mouvance du big data, héritières du courant plus ancien des Environnements informatiques pour l'apprentissage humain (EIAH) et ses tentatives de modélisation. En somme, l'alliance d'une IA symbolique et d'une IA connexionniste, davantage que le passage de l'une à l'autre.

\section{Un séminaire dans la continuité du SIF}

39 Pour avancer sur l'ensemble de ces questions, nous voudrions au sein du GIS2IF mettre en place un nouveau séminaire, s'inscrivant dans la continuité des travaux du Séminaire Industrialisation de la Formation (SIF).

Comme nous l'avons vu, l'IA relance aujourd'hui la question de l'industrialisation de la formation en promettant de rendre techniquement possible ce qui n'était qu'un rêve. Elle permettrait ainsi de passer d'une technologisation additive qui ne met pas des machines à la place des enseignants, professionnels de l'orientation, documentalistes ou autres personnels à une technologisation substitutive, au moins partielle, notamment pour les tâches jugées les moins créatives. Il s'agirait alors, selon Cédric Villani (2018, p.186) de "développer une complémentarité capacitante avec l'IA en développant la place de la créativité dans l'enseignement ».

41 Cette substitution - au moins partielle - ouvrirait la voie, selon Villani, au développement de nouvelles capacités d'action des enseignants. Le temps de travail libéré permettrait par exemple aux enseignants de documenter et de partager leurs pratiques pédagogiques.

42 Ces questions ont été précisément étudiées pendant plusieurs décennies par un courant de recherche portant sur l'industrialisation de la formation, créateur du séminaire éponyme ancré dans les sciences de l'information et de la communication et les sciences de l'éducation, dont les travaux ont donné lieu à de nombreuses publications dont l'anthologie commentée de textes sur l'industrialisation de 1913 à 2012 (Mœglin, dir., 2016). 

suivante: dans quelle mesure l'IA est-elle susceptible de rénover un projet d'industrialisation de la formation, déjà ancien et partiellement mis en œuvre, selon des voies qu'il s'agirait de repenser? Ce projet mettrait davantage encore que d'autres courants avant lui le processus de technologisation en avant, susceptible d'apporter aujourd'hui ou dans un futur proche des réponses inédites à de vieilles questions, comme celle de l'individualisation dans des systèmes d'enseignement très largement massifiés. Il s'agirait également d'interroger un projet industrialisant qui met en avant à la fois une logique de standardisation tout en prônant le développement de la créativité à tous les niveaux du système éducatif. Cette double orientation, qui pourrait sembler contradictoire, est en phase avec ce que l'on observe depuis plusieurs années dans le champ des industries de la culture et de la communication, en passe de se transformer en industries dites créatives (Bouquillion, Miège, Mœglin, 2013).

\section{BIBLIOGRAPHIE}

\section{Bibliographie}

Chris Anderson (2019). « La fin de la théorie. Le déluge de data rend la méthode scientifique obsolète ", traduit de l'anglais par Pierre-Emmanuel Dauzat, Le Débat, 2019/5, n 207, pp. 119-122.

Daniel Andler (2016). La silhouette de l'humain. Quelle place pour le naturalisme dans le monde d'aujourd'hui ?, Paris, Gallimard.

Bruno Bachimont (2015). «Le numérique comme milieu : enjeux épistémologiques et phénoménologiques ", Interfaces numériques, volume 4 - n³/2015, pp. 385-402.

Catherine Becchetti-Bizot, Guillaume Houzel et François Taddei (2017). « Vers une société apprenante : rapport sur la recherche et développement de l'éducation tout au long de la vie ", rapport remis à Najat Vallaud-Belkacem.

Philippe Bouquillion, Bernard Miège, Pierre Mœglin (2013). L'industrialisation des biens symboliques. Les industries créatives en regard des industries culturelles, Grenoble, PUG.

Dominique Cardon, Jean-Philippe Cointet, Antoine Mazières (2018). « La revanche des neurones. L'invention des machines inductives et la controverses de l'intelligence artificielle ", Réseaux, 2018/5, n² 211, pp. 173-220. https://www.cairn.info/revue-reseaux-2018-5-page-173.htm

Alain Desrosières (2014). Prouver et gouverner : Une analyse politique des statistiques publiques, La Découverte, 2014.

Emile Durkheim (1981, $1^{\text {re }}$ édition 1895). Les règles de la méthode sociologique, Paris, PUF.

Emile Durkheim (2013, $1^{\text {re }}$ édition 1897). Le suicide, Paris, PUF.

Yves Jeanneret (2014). Critique de la trivialité. Les médiations de la communication, enjeu de pouvoir, Paris, Éditions non standard.

Communication, technologies et développement, $10 \mid 2021$ 
Michael Jordan (2019). « Artificial Intelligence : The Revolution hasn't happened yet », Harvard Data Science Review, Issue 1.1, summer 2019.

Brahim Lamine, Laurent Petit (2014). « Les boîtiers de réponse pour un apprentissage interactif en amphithéâtre. Une expérience d'accompagnement et d'évaluation par la recherche », in Geneviève Lameul, Catherine Loisy, dir. (2014). La pédagogie universitaire à l'heure du numérique, Bruxelles, De Boeck Université.

Ronan Le Roux (2018). Une histoire de la cybernétique en France (1948-1975), Paris, Classiques Garnier.

Pierre Lévy (1994). L'intelligence collective. Pour une anthropologie du cyberespace, Paris, La Découverte.

Antoine Mazières (2016). Cartographie de l'apprentissage artificiel et de ses algorithmes, thèse de doctorat, Université Paris Diderot.

Pierre-Michel Menger, Simon PAYE, dir. (2017). Big Data et traçabilité numérique. Les sciences sociales face à la quantification massive des individus, Paris, Collège de France.

Robert King Merton (1948). «The self-fulfilling prophecy », The Antioch Review, vol. 8, n², pp. 193-210.

Pierre Mœglin (1994). Le satellite éducatif. Média et expérimentation, Paris, CNET, Collection « Réseaux ».

Pierre Mœglin (2010). Les industries éducatives, Paris, PUF, Collection « Que sais-je ?».

Pierre Mœglin, dir. (2016). Industrialiser l'éducation. Anthologie commentée (1913-2012), Saint-Denis, Presses universitaires de Vincennes.

Frank Pasquale (2015). Black Box Society, Les algorithmes secrets qui contrôlent l'économie et l'information, Limoges, Fyp Éditions.

Jean-Claude Passeron (2001). «La forme des preuves dans les sciences historiques », Revue européenne des sciences sociales, XXXIX-120 (2001).

Daniel Peraya et Vanda Luengo (2019). « Les Learning analytics vus par Vanda Luengo. Entretien », Distances et Médiations des savoirs, 27/2019.

Laurent Petit (2018). « Forme scolaire et évolutions paradigmatiques », Distances et Médiations des Savoirs, $n^{\circ} 24 / 2018$, https://journals.openedition.org/dms/3233

Antoinette Rouvroy, Thomas Berns (2013). « Gouvernementalité algorithmique et perspectives d'émancipation. Le disparate comme condition d'individuation par la relation? ", Réseaux, 2013/1, n 177, pp. 163-196.

Alain Supiot (2015). La gouvernance par les nombres, Cours au Collège de France (2012-2014), Paris, Fayard, Poids et Mesures du monde.

Alain Supiot (2016). « Quand les nombres nous gouvernent. Entretien avec Alain Supiot », Etudes, 2016/9 septembre, pp. 53-66.

Jean-Sébastien Vayre (2018). « Les machines apprenantes et la (re)production de la société : les enjeux communicationnels de la socialisation algorithmique? ", Les Enjeux de l'information et de la communication, $\mathrm{n}^{\circ}$ 19/2, pp. 93-111.

Cédric Villani (2018). Donner un sens à l'intelligence artificielle. Pour une stratégie nationale et européenne, Rapport de mission parlementaire.

Norbert Wiener (1948). Cybernetics or Control and Communication in the Animal and the Machine, Paris, Hermann, New York, J. Wiley \& Sons, Cambridge (Ma), Technology Press. Trad. Fr. R. Le 
Roux, N. Vallée-Lévi, R. Vallée (2014). La cybernétique. Information et régulation dans l'organisme et la machine, Paris, Editions du Seuil.

\section{NOTES}

1. La cybernétique ou science du gouvernement des systèmes est un néologisme attribué à Norbert Wiener (1948).

2. Nous n'ignorons pas les controverses qui entourent les recherches en filiation de plusieurs courants de recherche contemporains vis-à-vis de la cybernétique. Cardon, Cointet et Mazières (op. cit.) s'appuient sur les travaux de Dupuy sur les origines des sciences cognitives pour assimiler la première cybernétique au connexionnisme. Or, Le Roux dans sa critique de l'ouvrage de Dupuy (2005) y voit un souci de légitimation de la filiation des sciences cognitives en cherchant à définir un connexionnisme « canal historique ».

3. Il s'appuie explicitement sur les travaux d'histoire critique des statistiques menés par Alain Desrosières, voir notamment Alain Desrosières (2014). Prouver et gouverner : Une analyse politique des statistiques publiques, La Découverte.

4. Traduction française (2019). Dans son article, Anderson appuie significativement sa " démonstration » sur la physique et la biologie, deux disciplines expérimentales dans lesquelles la notion de modèle n'a pas du tout le même sens.

5. Ne pourrait-on parler davantage de "gouvernance algorithmique » en intégrant les analyses pertinentes de Supiot? Rouvroy et Berns (2013, p. 183) disent eux-mêmes que «ce gouvernement algorithmique approfondit encore l'idéal libéral d'une apparente disparition du projet même de gouverner ».

6. Durkheim a distingué quatre types de suicide : le suicide égoïste, le suicide altruiste, le suicide anomique et le suicide fataliste, mais il ne s'est guère attardé sur le dernier qui n'est évoqué que dans une note de bas de page.

7. Éric Dagiral et Sylvain Paraisie (in Menger, Paye, 2017) relativisent cette rupture épistémologique en montrant que dans les mondes sociaux dans lesquels elle se diffuse, la science des données, en raison de sa plasticité, s'adapte au contraire parfaitement aux différentes « épistémologies locales ", sans jamais les uniformiser.

8. http://www.nosignificantdifference.org/

9. Les chiffres de l'abandon en enseignement à distance ne circulent pas facilement car ils sont difficiles à admettre pour les institutions, voire participent de leur modèle économique. Sur cette question voir Mœglin (2010).

10. Toute ressemblance avec le projet porté par les plateformes américaines de MOOC de type Coursera, Udacity ou EdX à partir de 2012 n'est pas purement fortuite...

11. C'est nous qui transférons le raisonnement à la pédagogie, cette application n'est pas faite par les auteurs cités.

12. Cité par Bachimont (2015, p. 14).

13. Mazières (1996) distingue, outre le réseau de neurones artificiels, les arbres de décisions et forêts aléatoires, les réseaux bayésiens, la programmation génétique, la machine à vecteurs de support. 


\section{RÉSUMÉS}

Emballement médiatique aidant, les progrès de l'Intelligence artificielle (IA), s'appuyant sur un double phénomène de naturalisation des phénomènes sociaux et de fétichisation du nombre, soulèvent des enjeux épistémologiques majeurs pour les sciences humaines et sociales (SHS), les sciences de l'information et de la communication (SIC) en particulier. Le cas de la formation rend compte de la nature des défis à relever et permet d'esquisser des pistes de réponses possibles passant par un partenariat interdisciplinaire équilibré.

With the help of media hype, the progress of Artificial Intelligence (AI), based on a double phenomenon of naturalization of social phenomena and fetishization of numbers, raises major epistemological issues for Human and Social Sciences (HSS), and Information and Communication Sciences (ICS) in particular. The case of training illustrates the nature of the challenges to be met and allows us to sketch out possible answers through a balanced interdisciplinary partnership.

Con el bombo y platillo de los medios de comunicación, el progreso de la Inteligencia Artificial (IA), basado en un doble fenómeno de naturalización de los fenómenos sociales y de fetichización del número, plantea importantes cuestiones epistemológicas para las Ciencias Humanas y Sociales (SHS), las Ciencias de la Información y de la Comunicación (SCI) en particular. El caso de la formación ilustra la naturaleza de los retos a los que hay que hacer frente y nos permite esbozar posibles respuestas mediante una asociación interdisciplinar equilibrada.

\section{INDEX}

Mots-clés : Intelligence artificielle (IA), enjeux épistémologiques, individualisation de la formation.

Palabras claves : Inteligencia artificial (IA), cuestiones epistemológicas, individualización de la formación.

Keywords : Artificial intelligence (AI), epistemological issues, individualization of education.

\section{AUTEUR}

LAURENT PETIT

Sorbonne université 